\title{
Asymptotic Tracking of Second-Order Nonsmooth Feedback Stabilizable Unknown Systems with Prescribed Transient Response
}

\author{
Christos K. Verginis, Member, IEEE, and Dimos V. Dimarogonas, Senior Member, IEEE,
}

\begin{abstract}
This paper considers the asymptotic tracking control problem for a class of nonlinear systems subject to predefined constraints for the system response, such as maximum overshoot or minimum convergence rate. In particular, by employing discontinuous control protocols and nonsmooth analysis, we extend previous results on funnel control to guarantee at the same time asymptotic trajectory tracking. We consider 2ndorder systems that are affine in the control input and contain completely unknown nonlinear and nonsmooth vector fields, with no boundedness or approximation/parametric factorization assumptions. Simulation results verify the theoretical findings.
\end{abstract}

Index Terms-Uncertain systems, Robust adaptive control, Asymptotic stability, funnel control, prescribed performance control, nonlinear systems, non-smooth systems.

\section{INTRODUCTION}

C ONTROL of uncertain systems is one of the main research topics in systems theory. Robust and adaptive control as well as neural network/fuzzy logic control are the dominant methodologies dealing with such systems [1], [2]. There exists a variety of works achieving both asymptotic and "practical" (ultimately bounded errors) stability under the presence of model uncertainties (e.g., [3]-[12]). The majority of the related works that achieve asymptotic stability assume parametric dynamic uncertainties and/or growth conditions and gain tuning [4], local Neural network approximations [11], or fuzzy logic controllers [12].

A well-studied special instance of adaptive control is funnel control, where the output of the system is confined to a predefined funnel [13]-[17]. It is a model-free control scheme of high-gain type, with numerous applications during the last years. Examples include electrical circuits [18], Lagrangian systems [19]-[21] and multi-agent systems [21]-[23]. The intuition behind funnel control is the incorporation of an adaptive gain in the control scheme, which increases (in absolute value) as the system's output reaches the funnel's boundary. In that way, the system's output is "pushed" to always remain inside the funnel. Funnel control has been developed for both linear [18] and nonlinear systems [13] involving parametric [19] as well as structural [13], [14] dynamic uncertainties.

The authors are with the School of Electrical Engineering and Computer Science, KTH Royal Institute of Technology, SE-100 44, Stockholm, Sweden. Email: $\{$ cverginis, dimos\}akth.se. This work was supported by the H2020 ERC Starting Grant BUCOPHSYS, the European Union's Horizon 2020 Research and Innovation Programme under the GA No. 731869 (Co4Robots), the Swedish Research Council (VR), the Knut och Alice Wallenberg Foundation (KAW) and the Swedish Foundation for Strategic Research (SSF)
An important property that most related funnel-control works fail to achieve is that of asymptotic stability subject to unknown nonlinear dynamics. Traditional funnel control guarantees only confinement of the system output in a prespecified funnel, and thus the closest property to asymptotic stability that can be achieved is that of "practical stability", where the funnel converges arbitrarily close to zero. The latter, however, might yield undesired large inputs due to the small funnel values, and can be problematic in real-time systems. Such a scheme was developed in the works [24], [25] for first-order systems, where the funnel converges to zero. This, however, can create numerical ill-conditioning in the practical computation of the control input, since it involves the product of "large" and "small" quantities (the funnel reciprocal and and the error signal) [25]. On the other hand, with potential guarantees of asymptotic stability, the funnel is not needed to converge close to zero, and can be used in order to encode just transient constraints for the system. Asymptotic tracking subject to transient constraints has been considered in several works [19], [22], [26], [27]; [22], [26], [27] consider linear systems (LTI and double integrator), whereas [19] assumes known model structure, with the uncertainties being only parametric; One can conclude that the aforementioned works cannot be extended in a straightforward manner to nonlinear systems where the dynamic terms have both parametric and structural uncertainties. In addition, a class of systems for which funnel control has not been taken into account in the related works is the non-smooth type, i.e., systems with discontinuous right-hand side. Such models are motivated by real-time systems, where several dynamic terms (e.g., friction) can be accurately modeled by discontinuous state functions.

In this paper, we consider the asymptotic tracking control problem subject to transient constraints imposed by a predefined funnel. We consider MIMO systems of the form

$$
\begin{aligned}
\dot{x}_{1} & =x_{2}, \\
\dot{x}_{2} & =F(x, z, t)+G(x, z, t) u, \quad y=x_{1} \\
\dot{z} & =F_{z}(x, z, t),
\end{aligned}
$$

where $z \in \mathbb{R}^{n_{z}}, x:=\left[x_{1}^{\top}, x_{2}^{\top}\right]^{\top} \in \mathbb{R}^{2 n}$, with $x_{j}:=$ $\left[x_{j_{1}}, \ldots, x_{j_{n}}\right]^{\top} \in \mathbb{R}^{n}, \forall j \in\{1,2\}$, are the system's states, $y:=\left[y_{1}, \ldots, y_{n}\right]^{\top} \in \mathbb{R}^{n}$ is the system's output, which is required to track a desired trajectory $y_{\mathrm{d}}(t)$, and $F$ : $\mathbb{R}^{2 n+n_{z}} \times\left[t_{0}, \infty\right) \rightarrow \mathbb{R}^{n}, F_{z}: \mathbb{R}^{2 n+n_{z}} \times\left[t_{0}, \infty\right) \rightarrow \mathbb{R}^{n_{z}}$ $G: \mathbb{R}^{2 n+n_{z}} \times\left[t_{0}, \infty\right) \rightarrow \mathbb{R}^{n \times n}$ are unknown vector fields, not necessarily continuous. We assume that $x$ is available 
for measurement, whereas $z$ is not. In fact, the dynamics governing $z$ is called dynamic uncertainty and represents unmodeled dynamic phenomena that potentially affect the closed-loop response. The assumptions on the system dynamics are restricted to local boundedness and measurability as well as controllability conditions on $G$ and internal stability of $z$, without considering any uniform boundedness/growth condition or model approximation:

Assumption 1: The maps $(x, z) \mapsto F(x, z, t): \mathbb{R}^{2 n+n_{z}} \rightarrow$ $\mathbb{R}^{n},(x, z) \mapsto G(x, z, t): \mathbb{R}^{2 n+n_{z}} \rightarrow \mathbb{R}^{n \times n},(x, z) \mapsto$ $F_{z}(x, z, t): \mathbb{R}^{2 n+n_{z}} \rightarrow \mathbb{R}^{n_{z}}$ are Lebesgue measurable and locally bounded for each fixed $t \in\left[t_{0}, \infty\right)$, uniformly in $t$, and the maps $t \mapsto F(x, z, t):\left[t_{0}, \infty\right) \rightarrow \mathbb{R}^{n}$ and $t \mapsto G(x, z, t):\left[t_{0}, \infty\right) \rightarrow \mathbb{R}^{n \times n}$ are Lebesgue measurable and uniformly bounded for each fixed $(x, z) \in \mathbb{R}^{2 n+n_{z}}$, by unknown bounds.

Assumption 2: The matrix

$$
\widetilde{G}(x, z, t):=G(x, z, t)+G(x, z, t)^{\top}
$$

is positive definite, $\forall(x, z, t) \in \mathbb{R}^{2 n+n_{z}} \times\left[t_{0}, \infty\right)$, i.e., it holds that $\lambda_{\min }(\widetilde{G}(x, z, t))>0$, where $\lambda_{\min }(\widetilde{G}(x, z, t))$ is its unknown minimum eigenvalue.

Assumption 3: There exists a sufficiently smooth function $U_{z}: \mathbb{R}^{n_{z}} \rightarrow \mathbb{R}_{\geq 0}$ and class $\mathcal{K}_{\infty}$ functions $\gamma_{z}(\cdot), \bar{\gamma}_{z}(\cdot), \gamma_{z}(\cdot)$ such that $\underline{\gamma}_{z}(\|\bar{z}\|) \leq U_{z}(z) \leq \bar{\gamma}_{z}(\|z\|)$, and

$$
\left(\frac{\partial U_{z}}{\partial z}\right)^{\top} F_{z}(x, z, t) \leq-\gamma_{z}(\|z\|)+\pi_{z}(x, z, t)
$$

where $x \mapsto \pi_{z}(x, z, t): \mathbb{R}^{2 n} \rightarrow \mathbb{R}_{\geq 0}$ is continuous and class $\mathcal{K}_{\infty}$ for each fixed $(z, t) \in \mathbb{R}^{n_{z}} \times\left[t_{0}, \infty\right)$, and $(z, t) \mapsto$ $\pi_{z}(x, z, t): \mathbb{R}^{n_{z}} \times\left[t_{0}, \infty\right) \rightarrow \mathbb{R}_{\geq 0}$ is uniformly bounded for each fixed $x \in \mathbb{R}^{2 n}$.

Assumption 4: The state $x$ is available for measurement.

Assumption 5: The desired trajectory and its derivatives are bounded by finite and unknown constants $\bar{y}_{\mathrm{d}, 0}, \bar{y}_{\mathrm{d}, 1}>0$, i.e., $\left\|y_{d}(t)\right\|<\bar{y}_{\mathrm{d}, 0} \leq \bar{y}_{\mathrm{d}},\left\|y_{d}(t)\right\|<\bar{y}_{\mathrm{d}, 1} \leq \bar{y}_{\mathrm{d}}, \forall t \in\left[t_{0}, \infty\right)$, where $\bar{y}_{\mathrm{d}}:=\max _{j \in\{0,1\}}\left\{\bar{y}_{\mathrm{d}, j}\right\}$.

Note that Assumption 2 is a sufficient controllability condition and Assumption 3 suggests that $z$ is input-to-state practically stable with respect to $x, z, t$ implying stable zero (internal) dynamics [13]. Note also that the vector fields $F(\cdot)$ and $G(\cdot)$ are not required to be continuous everywhere.

According to the authors' best knowledge, this is the first work that guarantees asymptotic stability for a 2nd-order system subject to funnel constraints and under the considered assumptions 1-5, from all initial conditions that are compliant with the funnel and independent of the system model. In fact, according to the authors' best knowledge, asymptotic stability (even without funnel constraints) for 2nd-order systems has not been guaranteed in the related literature under these assumptions (i.e., unknown nonlinear discontinuous dynamics and no boundedness/growth assumptions on $F(\cdot)$ ) and from initial conditions independent of the system model. Regarding the latter, note that, if the initial value of the funnel is a design parameter, it can be always set larger than the initial value of the error to be confined in the funnel, rendering thus the results global. This work extends our preliminary results [28], which considered the special instance of time-invariant 2nd-order Lagrangian systems, to systems with more general dynamic terms $F, G$ and relaxation of the positive definitiveness of $G$.

The rest of the paper is structured as follows. Section II introduces some preliminary background and the notation followed throughout the article. Section III provides the proposed control protocol as well as the corresponding stability analysis. Finally, simulation results are given in Section IV and Section $\mathrm{V}$ concludes the paper.

\section{Notation AND PRELIMINARIES}

\section{A. Notation}

The sets of real and positive real numbers are denoted by $\mathbb{R}$ and $\mathbb{R}_{>0}$, respectively; $\|x\|$ denotes the 2 -norm of a vector $x \in \mathbb{R}^{n}$; The open and closed balls with respect to the 2 -norm and with radius $\delta$, centered at $x \in \mathbb{R}^{n}$, are denoted by $\mathcal{B}(x, \delta)$ and $\overline{\mathcal{B}}(x, \delta)$, respectively.

\section{B. Nonsmooth Analysis}

Consider the following differential equation with a discontinuous right-hand side:

$$
\dot{x}=f(x, t),
$$

where $f: \mathcal{D} \times\left[t_{0}, \infty\right) \rightarrow \mathbb{R}^{n}, \mathcal{D} \subset \mathbb{R}^{n}$, is Lebesgue measurable and locally essentially bounded, uniformly in $t$. The Filippov regularization of $f$ is defined as [29]

$$
\mathrm{K}[f](x, t):=\bigcap_{\delta>0} \bigcap_{\mu(\bar{N})=0} \overline{\operatorname{co}}(f(\mathcal{B}(x, \delta) \backslash \bar{N}), t),
$$

where $\bigcap_{\mu(\bar{N})=0}$ is the intersection over all sets $\bar{N}$ of Lebesgue measure zero, and $\overline{\mathrm{co}}(E)$ is the convex closure of the set $E$.

Definition 1 (Def. 1 of [30]): A function $x:\left[t_{0}, t_{1}\right) \rightarrow \mathbb{R}^{n}$, with $t_{1}>t_{0}$, is called a Filippov solution of (2) on $\left[t_{0}, t_{1}\right)$ if $x(t)$ is absolutely continuous and if, for almost all $t \in\left[t_{0}, t_{1}\right)$, it satisfies $\dot{x} \in \mathrm{K}[f](x, t)$, where $\mathrm{K}[f](x, t)$ is the Filippov regularization of $f(x, t)$.

Lemma 1 (Lemma 1 of [30]): Let $x(t)$ be a Filippov solution of (2) and $V: \mathcal{D} \times\left[t_{0}, t_{1}\right) \rightarrow \mathbb{R}$ be a locally Lipschitz, regular function ${ }^{1}$. Then $V(x(t), t)$ is absolutely continuous, $\dot{V}(x(t), t)=\frac{\partial}{\partial t} V(x(t), t)$ exists almost everywhere (a.e.), i.e., for almost all $t \in\left[t_{0}, t_{1}\right)$, and $\dot{V}(x(t), t) \stackrel{\text { a.e }}{\in} \dot{\widetilde{V}}(x(t), t)$, where

$$
\dot{\tilde{V}}:=\bigcap_{\xi \in \partial V(x, t)} \xi^{\top}\left[\begin{array}{c}
\mathrm{K}[f](x, t) \\
1
\end{array}\right],
$$

and $\partial V(x, t)$ is Clarke's generalized gradient at $(x, t)$ [30].

Theorem 1 (Corollary 2 of [30]): For the system given in (2), let $\mathcal{D} \subset \mathbb{R}^{n}$ be an open and connected set containing $x=0$ and suppose that $f$ is Lebesgue measurable and $x \mapsto$ $f(x, t)$ is essentially locally bounded, uniformly in $t$. Let $V$ : $\mathcal{D} \times\left[t_{0}, t_{1}\right) \rightarrow \mathbb{R}$ be locally Lipschitz and regular such that $W_{1}(x) \leq V(x, t) \leq W_{2}(x), \forall t \in\left[t_{0}, t_{1}\right), x \in \mathcal{D}$, and

$$
z \leq-W(x(t)), \quad \forall z \in \dot{\tilde{V}}(x(t), t), t \in\left[t_{0}, t_{1}\right), x \in \mathcal{D},
$$

${ }^{1}$ See [30] for a definition of regular functions. 
where $W_{1}$ and $W_{2}$ are continuous positive definite functions and $W$ is a continuous positive semi-definite on $\mathcal{D}$. Choose $r>0$ and $c>0$ such that $\overline{\mathcal{B}}(0, r) \subset \mathcal{D}$ and $c<$ $\min _{\|x\|=r} W_{1}(x)$. Then for all Filippov solutions $x:\left[t_{0}, t_{1}\right) \rightarrow$ $\mathbb{R}^{n}$ of (2), with $x\left(t_{0}\right) \in \mathbb{D}:=\left\{x \in \overline{\mathcal{B}}(0, r): W_{2}(x) \leq c\right\}$, it holds that $t_{1}=\infty, x(t) \in \mathbb{D}, \forall t \in\left[t_{0}, \infty\right)$, and $\lim _{t \rightarrow \infty} W(x(t))=0$.

\section{MAIN RESULTS}

The control objective is the asymptotic output tracking of a desired bounded trajectory $y_{\mathrm{d}}:=\left[y_{1, \mathrm{~d}}, \ldots, y_{n, \mathrm{~d}}\right]:\left[t_{0}, \infty\right) \rightarrow$ $\mathbb{R}^{n}$, with bounded derivatives, as stated in Assumption 5. Moreover, as discussed in Section I, we aim at imposing a certain predefined behavior for the transient response of the system. More specifically, motivated by funnel control techniques [14], [31], [32], given $n$ predefined funnels, described by the smooth functions (also called performance functions in [13]) $\rho_{p_{i}}:\left[t_{0}, \infty\right) \rightarrow\left[\underline{\rho}_{p_{i}}, \bar{\rho}_{p_{i}}\right] \subset \mathbb{R}_{>0}$, where $\underline{\rho}_{p_{i}}, \bar{\rho}_{p_{i}} \in \mathbb{R}_{>0}$ are positive lower and upper bounds, respectively, we aim at guaranteeing that ${ }^{2}-\rho_{p_{i}}(t)<y_{i}(t)-y_{i, \mathrm{~d}}(t)<\rho_{p_{i}}(t), \forall t \in$ $\left[t_{0}, \infty\right)$, given that $-\rho_{p_{i}}\left(t_{0}\right)<y_{i}\left(t_{0}\right)-y_{i, \mathrm{~d}}\left(t_{0}\right)<\rho_{p_{i}}\left(t_{0}\right)$, $\forall i \in\{1, \ldots, n\}$. These functions can encode maximum overshoot or convergence rate properties. Note that, compared to the majority of the related works on funnel control (e.g., [13], [14], [24], [31]), we do not require arbitrarily small final values $\lim _{t \rightarrow \infty} \rho_{p_{i}}(t)$, which would achieve convergence of $y_{i}(t)-y_{i, \mathrm{~d}}(t)$ arbitrarily close to zero, since one of the objectives is actual asymptotic stability. In this section, the problem statement is as follows:

Problem 1: Consider the system (1) and let a desired trajectory $y_{\mathrm{d}}:\left[t_{0}, \infty\right) \rightarrow \mathbb{R}^{n}$ as well as $n$ prescribed funnels, described by $\rho_{p_{i}}:\left[t_{0}, \infty\right) \rightarrow\left[\underline{\rho}_{p_{i}}, \bar{\rho}_{p_{i}}\right], \forall i \in\{1, \ldots, n\}$. Design a control protocol $u \in \mathbb{R}^{n}$ such that

1) $\lim _{t \rightarrow \infty}\left(y_{i}(t)-y_{i, \mathrm{~d}}(t)\right)=0$,

2) $-\rho_{p_{i}}(t)<y_{i}(t)-y_{i, \mathrm{~d}}(t)<\rho_{p_{i}}(t)$,

$\forall i \in\{1, \ldots, n\}, t \in\left[t_{0}, \infty\right)$, and all closed loop signals remain bounded.

Our solution to Problem 1 is based on the error transformation proposed in [13], which converts the constrained error behavior $-\rho_{p_{i}}(t)<y_{i}(t)-y_{i, \mathrm{~d}}(t)<\rho_{p_{i}}(t)$ to an unconstrained one. More specifically, we define the errors

$$
e_{p}:=\left[e_{p_{1}}, \ldots, e_{p_{n}}\right]^{\top}:=y-y_{\mathrm{d}},
$$

as well as the error transformations $\varepsilon_{p_{i}} \in \mathbb{R}$ according to:

$$
e_{p_{i}}=\rho_{p_{i}} T\left(\varepsilon_{p_{i}}\right), \forall i \in\{1, \ldots, n\},
$$

where $T: \mathbb{R} \rightarrow(-1,1)$ is a smooth, strictly increasing analytic function, with $T(0)=0$. Since $T$ is increasing, the inverse mapping $T^{-1}:(-1,1) \rightarrow \mathbb{R}$ is well-defined, and it holds that

$$
\lim _{\zeta \rightarrow-\infty} T(\zeta)=-1, \quad \lim _{\zeta \rightarrow+\infty} T(\zeta)=1
$$

and hence, if $\varepsilon_{p_{i}}$ remains bounded in a compact set, the desired funnel objective $-\rho_{p_{i}}(t)<e_{p_{i}}(t)<\rho_{p_{i}}(t)$ is achieved, $\forall i \in$ $\{1, \ldots, n\}$. We further require that

$$
|\zeta|<\left|\frac{\partial T^{-1}(\zeta)}{\partial \zeta} T^{-1}(\zeta)\right|, \quad \forall \zeta \in(-1,1) .
$$

A possible choice that satisfies the aforementioned specifications is $T(\zeta)=\frac{\exp (\zeta)-1}{\exp (\zeta)+1}$.

From (5), we obtain

$$
\varepsilon_{p_{i}}=T^{-1}\left(\frac{e_{p_{i}}}{\rho_{p_{i}}}\right),
$$

which, after differentiation, becomes

$$
\dot{\varepsilon}_{p_{i}}=\frac{r_{p_{i}}}{\rho_{p_{i}}}\left(x_{2_{i}}-\dot{y}_{i, \mathrm{~d}}-\frac{\dot{\rho}_{p_{i}} e_{p_{i}}}{\rho_{p_{i}}}\right),
$$

or, in stack vector form,

$$
\dot{\varepsilon}_{p}=r_{p} \rho_{p}^{-1}\left(x_{2}-\dot{y}_{\mathrm{d}}-\dot{\rho}_{p} \rho_{p}^{-1} e_{p}\right),
$$

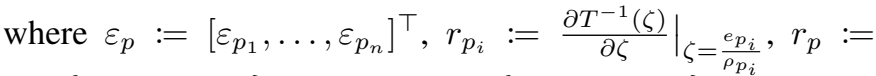
$\operatorname{diag}\left\{r_{p_{1}}, \ldots, r_{p_{n}}\right\}$, and $\rho_{p}:=\operatorname{diag}\left\{\rho_{p_{1}}, \ldots, \rho_{p_{n}}\right\}$. Due to the increasing property of $T(\cdot)$, it holds that $r_{p}$ is positive definite, and thus in order to render $\dot{\varepsilon}_{p}$ negative a straightforward choice for a desired value for $x_{2}$ is

$$
x_{2, \mathrm{~d}}:=\dot{y}_{\mathrm{d}}+\dot{\rho}_{p} \rho_{p}^{-1} e_{p}-k_{p} r_{p} \varepsilon_{p},
$$

where $k_{p} \in \mathbb{R}_{>0}$ is a positive and constant scalar gain. Since, however, $x_{2}$ is not the system's input, we follow a backstepping-like methodology and define the error

$$
e_{v}:=\left[e_{v_{1}}, \ldots, e_{v_{n}}\right]^{\top}:=x_{2}-x_{2, \mathrm{~d}} .
$$

Next, we proceed in a similar manner and define a funnel for each $e_{v_{i}}, i \in\{1, \ldots, n\}$, described by the functions $\rho_{v_{i}}:\left[t_{0}, \infty\right) \rightarrow\left[\underline{\rho}_{v_{i}}, \bar{\rho}_{v_{i}}\right] \subset \mathbb{R}_{>0}$, where $\underline{\rho}_{v_{i}}, \bar{\rho}_{v_{i}} \in \mathbb{R}_{>0}$ are the positive lower and upper bounds, respectively, with the constraint $\rho_{v_{i}}\left(t_{0}\right)>\left|e_{v_{i}}\left(t_{0}\right)\right|, i \in\{1, \ldots, n\}$. Note that $e_{v_{i}}\left(t_{0}\right)=x_{2}\left(t_{0}\right)-x_{2, \mathrm{~d}}\left(t_{0}\right)$ can be calculated at $t=t_{0}$ since it is a function of the state, the funnel functions and the desired trajectory profile. Then, we define the open set

$$
\begin{gathered}
\mathcal{D}_{u, t}:=\left\{(x, t) \in \mathbb{R}^{2 n} \times\left[t_{0}, \infty\right): \rho_{p}(t)^{-1} e_{p}(t) \in(-1,1)^{n},\right. \\
\left.\rho_{v}(t)^{-1} e_{v}(t) \in(-1,1)^{n}\right\},
\end{gathered}
$$

and design the control law $u: \mathcal{D}_{u, t} \rightarrow \mathbb{R}^{n}$ as

$$
u=-k_{v_{2}} \rho_{v}^{-1}\left(k_{v_{3}}\left\|r_{p} \varepsilon_{p}\right\|+k_{v_{4}} \hat{d}\right) s_{v}-k_{v_{1}} \rho_{v}^{-1} r_{v} \varepsilon_{v}
$$

where

$$
s_{v}:= \begin{cases}\frac{r_{v} \varepsilon_{v}}{\left\|r_{v} \varepsilon_{v}\right\|}, & \text { if }\left\|r_{v} \varepsilon_{v}\right\| \neq 0 \\ 0, & \text { otherwise, }\end{cases}
$$

$\rho_{v}:=\operatorname{diag}\left\{\rho_{v_{1}}, \ldots, \rho_{v_{n}}\right\}, \quad \varepsilon_{v}:=\left[\varepsilon_{v_{1}}, \ldots, \varepsilon_{v_{n}}\right]^{\top}, \quad \varepsilon_{v_{i}}:=$ $T^{-1}\left(\frac{e_{v_{i}}}{\rho_{v_{i}}}\right), r_{v}:=\operatorname{diag}\left\{r_{v_{1}}, \ldots, r_{v_{n}}\right\}, r_{v_{i}}:=\left.\frac{\partial T^{-1}(\zeta)}{\partial \zeta}\right|_{\zeta=\frac{e_{v_{i}}}{\rho_{v_{i}}}}$, $k_{v_{i}} \in \mathbb{R}_{>0}, i \in\{1, \ldots, 4\}$ are positive constant scalar gains, and $\hat{d}$ is an adaptive variable gain, subject to the constraint $\hat{d}\left(t_{0}\right) \geq 0$, and dynamics

$$
\dot{\hat{d}}=\gamma_{d}\left\|r_{v} \varepsilon_{v}\right\|
$$


where $\gamma_{d} \in \mathbb{R}_{>0}$ is a positive constant gain.

Remark 1: The control design procedure follows closely the prescribed performance backstepping-like methodology of the previous works [21], [23], [33], introduced in [34]. The desired signals and control laws in these works consist only of proportional terms with respect to the transformed errors $\varepsilon_{p}, \varepsilon_{v}$, i.e., $-k_{p} r_{p} \varepsilon_{p}$ and $-k_{v_{1}} \rho_{v}^{-1} r_{v} \varepsilon_{v}$ in (11) and (14), respectively, which are guaranteed to be ultimately bounded. In this work, we incorporate (a) the extra terms in (11) that would render (10) exponentially stable, and (b) the discontinuous term in (14), which, as will be shown in the sequel, enforces convergence of the transformed errors to zero, guaranteeing thus asymptotic stability. A similar discontinuous term was employed in [25] to achieve asymptotic stability, by enforcing however the funnel functions to converge to zero, which is not required in the proposed framework. Finally, we note that the adaptive term $\hat{d}=\gamma_{d} \int_{0}^{t}\left\|r_{v}(s) \varepsilon_{v}(s)\right\| d s$ in (14) is similar to the integrator term in PI-funnel control [16], introduced to attempt asymptotic stability, without, however, providing theoretical guarantees for the class of systems considered here.

Remark 2: Note that no information regarding the dynamic model is incorporated in the control protocol (4)-(15). All the necessary signals consist of the funnel terms $\rho_{p}, \rho_{v}$ and of known functions of the state and the desired trajectory $y_{\mathrm{d}}$. Furthermore, no a-priori gain tuning is needed and, as the next theorem states, the solution of Problem 1 is guaranteed from all initial conditions that satisfy $-\rho_{p_{i}}\left(t_{0}\right)<y_{i}\left(t_{0}\right)-y_{i, \mathrm{~d}}\left(t_{0}\right)<$ $\rho_{p_{i}}\left(t_{0}\right), \forall i \in\{1, \ldots, n\}$. As will be revealed subsequently, the adaptive gain $\hat{d}$ compensates the unknown dynamic terms, which are proven to be bounded due to the confinement of the state in the prescribed funnels.

The correctness of the control protocol (4)-(15) is shown in the next theorem.

Theorem 2: Consider a system subject to the dynamics (1), Assumptions 1-5, as well as a desired trajectory $y_{\mathrm{d}}$ and funnels as described in Problem 1 satisfying $-\rho_{p_{i}}\left(t_{0}\right)<y_{i}\left(t_{0}\right)-$ $y_{i, \mathrm{~d}}\left(t_{0}\right)<\rho_{p_{i}}\left(t_{0}\right), \forall i \in\{1, \ldots, n\}$. Then the control protocol (4)-(15) guarantees the existence of at least one local Filippov solution of the closed-loop system (1)-(14) that solves Problem 1. Moreover, every such local solution can be extended to a global solution and all closed-loop signals remain bounded, for all $t \geq t_{0}$.

Proof: The intuition of the subsequent proof is as follows: We first show the existence of at least one Filippov solution of the closed loop system in $\mathcal{D}_{u, t}$ for a time interval $I \subseteq\left[t_{0}, \infty\right)$. Next, we prove that for any of these solutions, the state remains bounded in $I$ by bounds independent of the endpoint of $I$. Hence, the dynamic terms of (1) are also upper bounded by a term, which we aim to compensate via the adaptation gain $\hat{d}$.

We start by defining some terms that will be used in the subsequent analysis: $M_{p}:=\max _{i \in\{1, \ldots, n\}}\left\{\bar{\rho}_{p_{i}}\right\}, m_{p}:=$ $\min _{i \in\{1, \ldots, n\}}\left\{\underline{\rho}_{p_{i}}\right\}, M_{\dot{p}}:=\max _{i \in\{1, \ldots, n\}}\left\{\sup _{t \geq t_{0}}\left\{\left|\dot{\rho}_{p_{i}}\right|\right\}\right\}$, $M_{v}:=\max _{i \in\{1, \ldots, n\}}\left\{\bar{\rho}_{v_{i}}\right\}, m_{v}:=\min _{i \in\{1, \ldots, n\}}\left\{\underline{\rho}_{v_{i}}\right\}$, $\underline{\lambda}:=\lambda_{\min }\left(\rho_{v}^{-1} \widetilde{G}(x, z, t) \rho_{v}^{-1}\right), \beta:=\left(k_{v_{2}} k_{v_{4}} \underline{\lambda}\right)^{-1}, \underline{r}_{p}:=$ $\inf _{\zeta \in(-1,1)} \frac{\partial T^{-1}(\zeta)}{\partial \zeta}$. Note that all the aforementioned terms are strictly positive. In particular, $\underline{\lambda}$ is strictly positive due to the definition of the funnels $\rho_{v}$ and Assumption 2, and $\underline{r}_{p}$ is strictly positive due to the strictly increasing property of $T(\cdot)$ and hence of $T^{-1}(\cdot)$. Moreover, in view of (6), it holds that $\arg \inf _{\zeta \in(-1,1)} \frac{\partial T^{-1}(\zeta)}{\partial \zeta} \in(-1,1)$.

By employing (14), (15), we can write the closed loop system

$$
\begin{aligned}
& \dot{x}_{1}=x_{2}, \\
& \dot{z} \in \mathrm{K}\left[F_{z}\right](x, z, t), \\
& \dot{x}_{2} \in \mathrm{K}[F](x, z, t)+\mathrm{K}[G](x, z, t) \mathrm{K}[u](x, t), \\
& \dot{\hat{d}}=\gamma_{d}\left\|r_{v} \varepsilon_{v}\right\|,
\end{aligned}
$$

where $\mathrm{K}[F](x, z, t), \mathrm{K}[G](x, z, t), \mathrm{K}[u](x, t)$ are the Filippov regularizations (see (3)) of the respective terms. For $u$ specifically, $\mathrm{K}[u](x, t)$ is formed by substituting the term $s_{v}$ with its reguralized term, which is $\mathrm{S}_{v}=\frac{r_{v} \varepsilon_{v}}{\left\|r_{v} \varepsilon_{v}\right\|}$ if $\left\|r_{v} \varepsilon_{v}\right\| \neq 0$, and $\mathrm{S}_{v} \in(-1,1)^{n}$ otherwise. Note that, in any case, it holds that $\left(r_{v} \varepsilon_{v}\right)^{\top} \mathrm{S}_{v}=\left\|r_{v} \varepsilon_{v}\right\|$. Define now $\widetilde{x}:=\left[x^{\top}, z^{\top}, \hat{d}\right] \in \mathbb{R}^{2 n+n_{z}+1}$ and consider the open set $\mathcal{D}_{c}:=\left\{(\widetilde{x}, t) \in \mathbb{R}^{2 n+n_{z}+1} \times\left[t_{0}, \infty\right):(x, t) \in \mathcal{D}_{u, t}\right\}$. Since $\rho_{p_{i}}\left(t_{0}\right)>\left|e_{p_{i}}\left(t_{0}\right)\right|$ and $\rho_{v_{i}}\left(t_{0}\right)>\left|e_{v_{i}}\left(t_{0}\right)\right|, \forall i \in\{1 \ldots, n\}$, the set $\mathcal{D}_{c}$ is nonempty. Moreover, since $T(\cdot)$, and hence its derivative, are analytic, their zero sets have zero measure [35] and thus the right hand-side of (16) is Lebesgue measurable and locally essentially bounded in $\widetilde{x}$ over the set $\left\{\widetilde{x}:(\widetilde{x}, t) \in \mathcal{D}_{c}\right\}$, and Lebesgue measurable in $t$ over the set $\left\{t:(\widetilde{x}, t) \in \mathcal{D}_{c}\right\}$. Hence, according to Prop. 3 of [36], for each initial condition $\left(\widetilde{x}\left(t_{0}\right), t_{0}\right) \in \mathcal{D}_{c}$, there exists at least one Filippov solution $\widetilde{x}(t)$ of (16), defined in $I:=\left[t_{0}, t_{\max }\right)$, where $t_{\text {max }}>t_{0}$ such that $(\widetilde{x}(t), t) \in \mathcal{D}_{c}, \forall t \in I$. By applying (8), we conclude the existence of the respective Filippov solutions $\varepsilon_{p}(t), \varepsilon_{v}(t) \in \mathbb{R}^{n}, \forall t \in I$. Let now $\widetilde{x}\left(t_{0}\right)$ denote the initial condition of the system (16) satisfying $\left(\widetilde{x}\left(t_{0}\right), t_{0}\right) \in \mathcal{D}_{c}$ and consider the family of Filippov solutions starting from $\widetilde{x}\left(t_{0}\right)$ denoted by the set $\mathfrak{X}$. Note that, although not explicitly stated, $t_{\text {max }}$ and $I$ might be different for each solution in $\mathfrak{X}$. We aim to prove that all $\varepsilon_{p}(t), \varepsilon_{v}(t)$ are bounded and converge to zero, for all $\widetilde{x}(t) \in \mathfrak{X}$.

In view of the definition of $\mathcal{D}_{c}$ (see also (13)), for all $\widetilde{x}(t) \in$ $\mathfrak{X}$ it holds that

$$
\left|e_{p_{i}}(t)\right|<\bar{\rho}_{p_{i}},\left|e_{v_{i}}(t)\right|<\bar{\rho}_{v_{i}}
$$

$\forall t \in I$, where $\bar{\rho}_{p_{i}}$ and $\bar{\rho}_{v_{i}}$ are the upper bounds of $\rho_{p_{i}}(t)$ and $\rho_{v_{i}}(t)$, respectively, $\forall i \in\{1, \ldots, n\}$. Consider now the 
Lyapunov function $V_{p}:=\frac{1}{2}\left\|\varepsilon_{p}\right\|^{2}$, for which it holds, in view of (10), (11), (12), and (17)

$$
\begin{aligned}
\dot{V}_{p} & =\varepsilon_{p}^{\top} r_{p} \rho_{p}^{-1}\left(x_{2}-\dot{y}_{\mathrm{d}}-\dot{\rho}_{p} \rho_{p}^{-1} e_{p}\right) \\
& =-k_{p} \varepsilon_{p}^{\top} r_{p} \rho_{p}^{-1} r_{p} \varepsilon_{p}+\varepsilon_{p}^{\top} r_{p} \rho_{p}^{-1} e_{v} \\
& <-\frac{k_{p}}{M_{p}}\left\|r_{p} \varepsilon_{p}\right\|^{2}+\frac{M_{v}}{m_{p}}\left\|r_{p} \varepsilon_{p}\right\|,
\end{aligned}
$$

$\forall t \in I$. Hence, we conclude that $\dot{V}_{p}<0$ when $\left\|r_{p} \varepsilon_{p}\right\|>$ $\frac{M_{v} M_{p}}{k_{p} m_{p}}$. Since $r_{p_{i}}$ is positive definite, $\forall i \in\{1, \ldots, n\}$, the latter is equivalent to $\left\|\varepsilon_{p}\right\|>\frac{M_{v} M_{p}}{k_{p} m_{p} \underline{\underline{r}}_{p}} \Rightarrow \dot{V}_{p}<0$. Hence, we conclude that all $\widetilde{x}(t) \in \mathfrak{X}$ satisfy

$$
\left\|\varepsilon_{p}(t)\right\| \leq \bar{\varepsilon}_{p}:=\max \left\{\left\|\varepsilon_{p}\left(t_{0}\right)\right\|, \frac{M_{v} M_{p}}{k_{p} m_{p} \underline{r}_{p}}\right\} .
$$

Since $\bar{\varepsilon}_{p}$ is finite, it holds that $T\left(\bar{\varepsilon}_{p}\right)<1$ and hence $\left|T\left(\varepsilon_{p_{i}}(t)\right)\right| \leq T\left(\bar{\varepsilon}_{p}\right)<1, \forall i \in\{1, \ldots, n\}, t \in I$. Moreover, since $T(\cdot)$ and $T^{-1}(\cdot)$ are smooth, the derivative $\frac{\partial T^{-1}(\zeta)}{\partial \zeta}$ approaches infinity only when $\zeta \rightarrow \pm 1$. Therefore, in view of the definition of $r_{p_{i}}$ in (10), we conclude the existence of a finite $\bar{r}_{p}>0$ such that $\left\|r_{p}(t)\right\| \leq \bar{r}_{p}, \forall t \in I$. Next, (5) implies that $\left\|e_{p}(t)\right\| \leq \bar{e}_{p}:=M_{p} T\left(\bar{\varepsilon}_{p}\right) \sqrt{n}, \forall t \in I$. Hence, we conclude that $\left\|x_{2, \mathrm{~d}}(t)\right\| \leq \bar{x}_{2, \mathrm{~d}}:=\bar{y}_{\mathrm{d}}+\frac{M_{\dot{p}}}{m_{p}} \bar{e}_{p}+k_{p} \bar{r}_{p} \bar{\varepsilon}_{p}, \forall t \in I$, where $\bar{y}_{\mathrm{d}}$ is the uniform bound of the desired trajectory, introduced in Assumption 5. We also conclude that $\left\|x_{1}(t)\right\| \leq \bar{x}_{1}:=\bar{e}_{p}+\bar{y}_{\mathrm{d}}$, $\forall t \in I$. In addition, by employing $x_{2}=e_{v}+x_{2, \mathrm{~d}}$ and (17), we conclude that $\left\|x_{2}(t)\right\|<\widetilde{x}_{2}:=M_{v} \sqrt{n}+\bar{x}_{2, \mathrm{~d}}, \forall t \in I$. Finally, by differentiating $x_{2, \mathrm{~d}}$, employing the smoothness and boundedness of $\rho_{p}$ and its derivatives, the smoothness of $T(\cdot)$, the boundedness of $\ddot{y}_{\mathrm{d}}(t)$ as well as the aforementioned bounds, we can conclude the existence of a bound $\bar{v}_{\mathrm{d}}$ such that $\left\|\dot{x}_{2, \mathrm{~d}}(t)\right\| \leq \bar{v}_{\mathrm{d}}, \forall t \in I$.

Furthermore, the boundedness of $x(t)$ and Assumption 3 imply the existence of a positive finite constant $\bar{z}$ such that $\|z(t)\| \leq \bar{z}, \forall t \in I$. Hence, since $F(x, z, t)$ is Lebesgue measurable and locally essentially bounded in $\mathbb{R}^{2 n+n_{z}}$ and $\left\|x_{1}(t)\right\| \leq \bar{x}_{1}<\infty,\left\|x_{2}(t)\right\|<\widetilde{x}_{2}<\infty$, $\|z(t)\| \leq \bar{z}, \forall t \in I$, there exists some positive $\bar{F}$, such that $\|F(x(t), z(t), t)\| \stackrel{a . e .}{\leq} \bar{F}, \forall t \in I$, and hence, for each $(x, z)$, since $\mathrm{K}[F]$ is formed by the convex closure of $F$, it holds that $\max _{\zeta \in \mathrm{K}[F](x(t), z(t), t)}\{\zeta\} \leq \bar{F}, \forall t \in I$ and $\widetilde{x}(t) \in \mathfrak{X}$. Note that, in view of the aforementioned discussion, $\bar{F}$ depends solely on the initial conditions and the parameters of the funnel functions. Define now the finite constant term $d \in \mathbb{R}_{>0}$ as

$$
d:=\frac{\beta}{m_{v}}\left(\bar{F}+\bar{v}_{\mathrm{d}}+M_{\dot{p}} \sqrt{n}\right) .
$$

Note that the term in the parenthesis of (18) is an upper bound for the term $\left\|F(x(t), z(t), t)-\dot{x}_{2, \mathrm{~d}}(t)-\dot{\rho}_{v}(t) \rho_{v}(t)^{-1} e_{v}(t)\right\|$, for all $\widetilde{x}(t) \in \mathfrak{X}$ and almost all $t \in I$.
Define also the signal $\widetilde{d}:=\hat{d}-d$, where $\hat{d}$ is the adaptive gain introduced in (14). Consider now the function

$$
V(\widetilde{\varepsilon}):=\alpha V_{p}+\frac{\beta}{2}\left\|\varepsilon_{v}\right\|^{2}+\frac{1}{2 \gamma_{\mathrm{d}}} \widetilde{d}^{2},
$$

where $\widetilde{\varepsilon}:=\left[\varepsilon_{p}^{\top}, \varepsilon_{v}^{\top}, \widetilde{d}\right]^{\top}$, and $\alpha>0$ is a positive constant to be defined; $V(\widetilde{\varepsilon})$ satisfies $W_{1}(\widetilde{\varepsilon}) \leq V(\widetilde{\varepsilon}) \leq W_{2}(\widetilde{\varepsilon})$, for $W_{1}(\widetilde{\varepsilon}):=$ $\min \left\{\frac{\alpha}{2}, \frac{\beta}{2}, \frac{1}{2 \gamma_{\mathrm{d}}}\right\}\|\widetilde{\varepsilon}\|^{2}$ and $W_{2}(\widetilde{\varepsilon}):=\max \left\{\frac{\alpha}{2}, \frac{\beta}{2}, \frac{1}{2 \gamma_{\mathrm{d}}}\right\}\|\widetilde{\varepsilon}\|^{2}$. Then, according to Lemma $1, \dot{V}(\widetilde{\varepsilon}(t)) \stackrel{\text { a.e. }}{\epsilon} \dot{\widetilde{V}}(\widetilde{\varepsilon}(t))$ with $\dot{\vec{V}}:=\bigcap_{\xi \in \partial V(\widetilde{\varepsilon})} \xi^{\top} \mathrm{K}[\dot{\tilde{\varepsilon}}]$. Since $V(\widetilde{\varepsilon})$ is continuously differentiable, its generalized gradient reduces to the standard gradient and thus it holds that $\dot{\tilde{V}}=\nabla V^{\top} \mathrm{K}[\dot{\tilde{\varepsilon}}]$, where $\nabla V=\left[\alpha \varepsilon_{p}^{\top}, \beta \varepsilon_{v}^{\top}, \frac{1}{\gamma_{\mathrm{d}}} \widetilde{d}\right]^{\top}$. After using (1), (14), (15), and $x_{2}=x_{2, \mathrm{~d}}+e_{v}$, one obtains

$$
\begin{aligned}
& \dot{\tilde{V}} \subset \widetilde{W}_{s}:=-\alpha k_{p} \varepsilon_{p}^{\top} r_{p} \rho_{p}^{-1} r_{p} \varepsilon_{p}+\alpha \varepsilon_{p}^{\top} r_{p} \rho_{p}^{-1} e_{v} \\
& -\beta k_{v_{1}} \varepsilon_{v}^{\top} r_{v} \rho_{v}^{-1} \mathrm{~K}[G](x, t) \rho_{v}^{-1} r_{v} \varepsilon_{v}+\widetilde{d}\left\|r_{v} \varepsilon_{v}\right\| \\
& +\beta \varepsilon_{v}^{\top} r_{v} \rho_{v}^{-1}\left(\mathrm{~K}[F](x, t)-\dot{x}_{2, \mathrm{~d}}-\dot{\rho}_{v} \rho_{v}^{-1} e_{v}\right) \\
& -\beta k_{v_{2}} \varepsilon_{v}^{\top} r_{v} \rho_{v}^{-1} \mathrm{~K}[G](x, t) \rho_{v}^{-1} \mathrm{~S}_{v}\left(k_{v_{3}}\left\|r_{p} \varepsilon_{p}\right\|+k_{v_{4}} \hat{d}\right) .
\end{aligned}
$$

Note that, since $\hat{d}\left(t_{0}\right) \geq 0,(15)$ implies that $\hat{d}(t) \geq 0, \forall t \in I$. Moreover, since the Filippov regularization (3) is defined as a closed set and $\dot{\tilde{V}} \subset \widetilde{W}_{s}$, it holds that $\max _{\zeta \in \dot{\tilde{V}}}\{\zeta\} \leq$ $\max _{\zeta \in \widetilde{W}_{s}}\{\zeta\}$. By substituting $G=\frac{G+G^{\top}}{2}+\frac{G-G^{\top}}{2}$ and employing the skew-symmetry of the second term, we obtain in view of Assumption 2 and the definition of $d$ in (18):

$$
\begin{aligned}
& \max _{\zeta \in \tilde{\widetilde{V}}}\{\zeta\} \leq \max _{\zeta \in \widetilde{W}_{s}}\{\zeta\} \leq-\alpha \frac{k_{p}}{M_{p}}\left\|r_{p} \varepsilon_{p}\right\|^{2}-k_{v_{1}} \beta \underline{\lambda}\left\|r_{v} \varepsilon_{v}\right\|^{2}- \\
& k_{v_{2}} k_{v_{4}} \beta \underline{\lambda}\left\|r_{v} \varepsilon_{v}\right\| \hat{d}-k_{v_{2}} k_{v_{3}} \beta \underline{\lambda}\left\|r_{v} \varepsilon_{v}\right\|\left\|r_{p} \varepsilon_{p}\right\|+\widetilde{d}\left\|r_{v} \varepsilon_{v}\right\| \\
& +\left\|r_{v} \varepsilon_{v}\right\| d+\alpha\left\|\varepsilon_{p}^{\top} r_{p} \rho_{p}^{-1} e_{v}\right\|,
\end{aligned}
$$

for all solutions $\widetilde{x}(t) \in \mathfrak{X}$. By setting $\zeta=T\left(\varepsilon_{v_{i}}\right)$ in (7), we obtain $\left|T\left(\varepsilon_{v_{i}}\right)\right| \leq\left|r_{v_{i}} \varepsilon_{v_{i}}\right|$ and hence by employing $e_{v_{i}}=$ $\rho_{v_{i}} T\left(\varepsilon_{v_{i}}\right), i \in\{1, \ldots, n\}$, we obtain that

$$
\alpha\left\|\varepsilon_{p}^{\top} r_{p} \rho_{p}^{-1} e_{v}\right\| \leq \alpha \frac{M_{v}}{m_{p}}\left\|r_{p} \varepsilon_{p}\right\|\left\|r_{v} \varepsilon_{v}\right\| .
$$

Therefore, by setting $\alpha=\frac{k_{v_{2}} k_{v_{3}} m_{p} \beta \underline{\underline{\lambda}}}{M_{v}}$, employing $d=\hat{d}-\widetilde{d}$, and in view of the fact that $\beta=\left(k_{v_{2}} k_{v_{4}} \underline{\lambda}\right)^{-1}$, we obtain

$$
\max _{\zeta \in \tilde{\tilde{V}}}\{\zeta\} \leq-\alpha \frac{k_{p}}{M_{p}}\left\|r_{p} \varepsilon_{p}\right\|^{2}-k_{v_{1}} \beta \underline{\lambda}\left\|r_{v} \varepsilon_{v}\right\|^{2}=:-W(\widetilde{\varepsilon}),
$$

$\forall t \in I, \widetilde{x}(t) \in \mathfrak{X}$, where $W$ is continuous and positive semidefinite on $\mathbb{R}^{2 n+1}$, since $r_{v}$ and $r_{p}$ are positive definite. Hence, we conclude that $\zeta \leq-W(\widetilde{\varepsilon}), \forall \zeta \in \dot{\widetilde{V}}(\widetilde{\varepsilon}(t)), \forall t \in I$ and all $\widetilde{x}(t) \in \mathfrak{X}$. Choose now any finite $r>0$ and let $c<$ 
$\min _{\|\widetilde{\varepsilon}\|=r} W_{1}(\widetilde{\varepsilon})$. Note that all the conditions of Theorem 1 are satisfied and hence, all Filippov solutions starting from $\widetilde{\varepsilon}\left(t_{0}\right) \in \Omega_{f}:=\left\{\widetilde{\varepsilon} \in \mathcal{B}(0, r): W_{2}(\widetilde{\varepsilon}) \leq c\right\}$ are bounded and remain in $\Omega_{f}, \forall t \in I$. Moreover, $t_{\max }=\infty$, implying that $I=\left[t_{0}, \infty\right)$ and it also holds that $\lim _{t \rightarrow \infty} W(\widetilde{\varepsilon})=0$, which, by invoking the increasing property of $T(\cdot)$ as well as the boundedness of $V$ (which imply the positive definiteness and boundedness of $\left.r_{p}, r_{v}\right)$, suggests that $\lim _{t \rightarrow \infty}\left\|\varepsilon_{p}(t)\right\|=0$ and $\lim _{t \rightarrow \infty}\left\|\varepsilon_{v}(t)\right\|=0$. The latter, in view of the increasing property of $T(\cdot)$ and the fact that $T(0)=0$, implies that $\lim _{t \rightarrow \infty}\left\|e_{p}(t)\right\|=0$ and $\lim _{t \rightarrow \infty}\left\|e_{v}(t)\right\|=0$.

Note that $r$, and hence $c$, can be arbitrarily large allowing any finite initial condition $\widetilde{\varepsilon}$, which implies any $\left(\widetilde{x}\left(t_{0}\right), t_{0}\right) \in \mathcal{D}_{c}$. In addition, it holds that $\|\widetilde{\varepsilon}\|^{2} \leq \widetilde{c}:=$ $\left(\max \left\{\frac{\alpha}{2}, \frac{\beta}{2}, \frac{1}{2 \gamma_{d}}\right\}\right)^{-1} c$, which implies the boundedness of $\left\|\varepsilon_{p}\right\|,\left\|\varepsilon_{v}\right\|$ and $\widetilde{d}$ by $\sqrt{\widetilde{c}}$. Therefore, we conclude that $\|\hat{d}(t)\| \leq$ $\bar{d}:=d+\sqrt{\widetilde{c}}, \forall t \in I$. Moreover, by employing (5), we conclude that $\left|\rho_{v_{i}}(t)^{-1} e_{v_{i}}(t)\right| \leq T(\sqrt{\widetilde{c}})<1$, and hence $\left|e_{v_{i}}(t)\right| \leq$ $M_{v} T(\sqrt{\widetilde{c}}) \Rightarrow\left\|x_{2}(t)\right\| \leq \bar{x}_{2}:=M_{v} T(\sqrt{\widetilde{c}}) \sqrt{n}+\bar{x}_{2, \mathrm{~d}}, \forall t \in I$. Therefore, we conclude that all solutions are bounded in compact sets $\forall t \in I$, which means that $u$, and $\dot{\hat{d}}$, as designed in (14) and (15), respectively, remain also bounded, $\forall t \in I$.

Remark 3: It is straightforward to apply the proposed methodology to the simpler case of first-order systems, i.e., when (1a) and (1b) are replaced by $\dot{x}=F(x, z, t)+$ $G(x, z, t) u$. The control law then takes the form $u=$ $-k_{2} \rho_{p}^{-1} \hat{d} s_{v}-k_{1} \rho_{p}^{-1} r_{p} \varepsilon_{p}$, with $\dot{\hat{d}}=\gamma_{d}\left\|r_{p} \varepsilon_{p}\right\|$, and $s_{v}$ defined using $r_{p} \varepsilon_{p}$. The boundedness of the solutions in the maximal interval of existence $I$ implies the boundedness of $F(\cdot)$ and $e_{p}$, and, by following a similar procedure with $V$ and $\dot{V}$, the derivative of $V_{p_{1}}:=\frac{\alpha_{1}}{2}\left\|\varepsilon_{p}\right\|^{2}+\frac{1}{2 \gamma_{d}} \widetilde{d}^{2}$ yields $\max _{\zeta \in \dot{V}_{p_{1}}}\{\zeta\} \leq-k_{3}\left\|r_{p} \varepsilon_{p}\right\|^{2}$ for some positive constants $\alpha_{1}$, $k_{3}$, implying $\lim _{t \rightarrow \infty}\left\|\varepsilon_{p}(t)\right\|=0$.

Remark 4: Note that no boundedness assumptions or growth conditions are needed for the vector fields $F(x, z, t)$ and $G(x, z, t)$. In particular, the effect of $F(x, z, t)$ is canceled by the introduced adaptive signal $\hat{d}$, which increases according to (15). It is proved, nevertheless, that this adaptive signal remains bounded. Moreover, the response of the system is solely determined by the funnel functions $\rho_{p_{i}}$ and $\rho_{v_{i}}$ (and $\rho_{s_{i}}$ in the second control scheme), isolated from the system dynamics and the control gains selection. Nevertheless, we note that appropriate gain tuning might be needed to suppress chattering in real life scenarios. Similarly, note that the region of attraction (initial conditions) of $\left(\varepsilon_{p}, \varepsilon_{v}\right)=(0,0)$ is independent from the system dynamics and the control gain selection and depends only on the choice of the funnel functions $\rho_{p_{i}}$, $\forall i \in\{1, \ldots, n\}$. In particular, if $\rho_{p_{i}}\left(t_{0}\right)$ are design parameters, we can always choose them such that $-\rho_{p_{i}}\left(t_{0}\right)<e_{p_{i}}\left(t_{0}\right)<$ $\rho_{p_{i}}\left(t_{0}\right), \forall i \in\{1, \ldots, n\}$, which renders the result global. In fact, the choice $\lim _{t \rightarrow t_{0}^{+}} \frac{1}{\rho_{p_{i}}\left(t_{0}\right)}=0, \forall i \in\{1, \ldots, n\}$ [14] is not excluded from our control scheme and does not restrict the initial condition $y\left(t_{0}\right)$. More specifically, one can redefine the transformed errors as $\varepsilon_{p_{i}}=T^{-1}\left(\phi_{p_{i}} e_{p_{i}}\right), \varepsilon_{v_{i}}=T^{-1}\left(\phi_{v_{i}} e_{v_{i}}\right)$, with $\phi_{p_{i}}, \phi_{v_{i}}$ being the reciprocals of the funnel boundaries satisfying $\phi_{p_{i}}\left(t_{0}\right)=\phi_{v_{i}}\left(t_{0}\right)=0$. The control law is then defined by replacing $\rho_{p_{i}}, \rho_{v_{i}}$ by $\phi_{p_{i}}^{-1}, \phi_{v_{i}}^{-1}$ in (14), $\forall i \in$ $\{1, \ldots, n\}$, and setting $x_{2, \mathrm{~d}}\left(t_{0}\right)=\dot{y}_{\mathrm{d}}-k_{p} r_{p} \varepsilon_{p}, x_{2, \mathrm{~d}}(t)=$ $\dot{y}_{\mathrm{d}}+\frac{\partial \phi_{p}^{-1}}{\partial t} \phi_{p} e_{p}-k_{p} r_{p} \varepsilon_{p}$, for $t>t_{0}$, since $\frac{\partial \phi_{p}^{-1}}{\partial t}$ is not defined at $t=t_{0}$. One can conclude that the aforementioned analysis is still valid and $\dot{V} \in \dot{\widetilde{V}}$ still holds for almost all $t \geq t_{0}$, implying $\lim _{t \rightarrow \infty}\left\|\varepsilon_{p}(t)\right\|=\lim _{t \rightarrow \infty}\left\|\varepsilon_{v}(t)\right\|=0$. Finally, noise can be taken into account in the measurement of $x_{2}$, i.e., consider that $x_{2}+n(x, t)$ is available for measurement, where $n(x, t)$ is an unknown noise signal with appropriate continuity and boundedness properties. By redefining $e_{v}=x_{2}+n(x, t)-x_{2, \mathrm{~d}}$ and including the time derivative of $n(x, t)$ in (18), the analysis still holds. Note, however, that in this case it can only be deduced that $\lim _{t \rightarrow \infty}\left(x_{2}(t)+n(x, t)-x_{2, \mathrm{~d}}(t)\right)=0$ and hence $x_{2}(t)$ does not necessarily converge to $x_{2, \mathrm{~d}}(t)$.

Remark 5: Since funnel control traditionally guarantees confinement of the state in the desired funnel, a common practice is to tune the funnel to converge to arbitrarily small values, achieving thus "practical stability", i.e., the state converging arbitrarily close to zero. Note that, in our case, the funnel functions $\rho_{p_{i}}, \rho_{v_{i}}$, are not required to decrease to values arbitrarily close to zero, yet asymptotic stability is still achieved. In fact, the proposed control schemes can be used to achieve merely asymptotic stability results without any funnel constraints, if the latter is not required. More specifically, given the initial errors $e_{p_{i}}\left(t_{0}\right)$, for two control schemes, respectively, we can use the proposed control protocols by employing any constant values $\rho_{p_{i}}>\left|e_{p_{i}}\left(t_{0}\right)\right|, \forall i \in\{1, \ldots, n\}$. Finally, the proposed control scheme can be extended to systems of the form $\dot{x}_{i}=\dot{x}_{i+1}, i \in\{1, \ldots, k-1\}, \dot{x}_{k}=F(x, z, t)+G(x, z, t) u$ for some $k>0$, where the funnel constraints are set for the combined signal $\sum_{j \in\{1, \ldots, k-1\}} e_{p}^{(k)}$.

\section{SIMULATION RESULTS}

We consider here the simulation of two inverted pendulum connected by a spring and a damper [13], with dynamics:

$$
\begin{aligned}
& J_{1} \ddot{x}_{1_{1}}=g_{s} \sin \left(x_{1_{1}}\right)-0.25 F_{s} \cos \left(x_{1_{1}}-\theta\right)-T_{1}+u_{1} \\
& J_{2} \ddot{x}_{1_{2}}=1.25 g_{s} \sin \left(x_{1_{2}}\right)+0.25 F_{s} \cos \left(x_{1_{2}}-\theta\right)-T_{2}+\sigma(t) u_{2},
\end{aligned}
$$

where $F_{s}:=150\left(d_{s}-0.5\right)+\dot{d}_{s}$ is the force between the connection points of the spring and damper, and

$$
d_{s}:=\sqrt{.25+.25\left(\sin \left(x_{1_{1}}-x_{1_{2}}\right)\right)+0.125\left(1-\cos \left(x_{1_{2}}-x_{1_{1}}\right)\right)}
$$


is the distance between these points; $\theta$ is defined as

$$
\theta:=\tan ^{-1}\left(\frac{0.25\left(\cos \left(x_{1_{2}}\right)-\cos \left(x_{1_{1}}\right)\right)}{0.5+0.25\left(\sin \left(x_{1}\right)-\sin \left(x_{2}\right)\right)}\right)
$$

and $T_{1}, T_{2}$ are friction terms on the motors evolving according to $T_{i}=d_{a_{i}}(t)+\tau_{i}+\dot{\tau}_{i}+\dot{x}_{1_{i}}$, with

$$
\dot{\tau}_{i}=\dot{x}_{1_{i}}-\frac{\left|\dot{x}_{1_{i}}\right|}{1+\exp \left(-\left|\frac{\dot{x}_{1_{i}}}{0.1}\right|^{2}\right)}
$$

and $d_{a_{i}}(t):=(-1)^{i-1} \cos (t)^{2}$, for $t \in\left[0, \frac{3 \pi}{2}\right) \cup\left[\frac{7 \pi}{2}, \frac{11 \pi}{2}\right) \cup$ $\left[\frac{21 \pi}{2}, \frac{27 \pi}{2}\right) \cup\left[\frac{35 \pi}{2}, 50\right)$ and 0 otherwise, $i \in\{1,2\}$ being an additional disturbance. The time varying signal $\sigma(t)$ is:

$$
\sigma(t)= \begin{cases}1 & \text { if } t \in[0,3) \cup[3.5, \infty) \\ 0.5 & \text { if } t \in[3,3.5)\end{cases}
$$

modeling a loss of effectiveness of the second motor when $t \in[3,3.5)$. We also choose $g_{s}=9.81$ as the gravity constant and $J_{1}=0.5, J_{2}=0.625$. The initial conditions are $t_{0}=0, x(0)=[0,0,0,0]^{\top}, \tau_{1}(0)=\tau_{2}(0)=0$ and the desired trajectory $y_{\mathrm{d}}=\left[2 \cos (t), \frac{\pi}{2}-2 \sin (t)\right]^{\top}$. The prescribed funnel functions are chosen as $\rho_{p_{i}}(t)=2.5 \exp (-0.1 t)+2.5$, $\forall i \in\{1,2\}$, which converge to 2.5 . We also choose $\rho_{v_{i}}(t)=$ $\left(\left\|e_{v}(0)\right\|_{1}-2\right) \exp (-0.1 t)+2.5$, as well as the gains $k_{p}=10$, $k_{v_{1}}=2 \cdot 10^{3}, k_{v_{2}}=0.1, k_{v_{3}}=0.025, k_{v_{4}}=0.05$, and $\gamma_{\mathrm{d}}=50$. The simulation results are depicted in Figs. 1-4 for $t \in[0,55]$ sec. More specifically, Fig. 1 depicts the errors $e_{p}(t), e_{v}(t)$ along with the performance functions $\rho_{p}(t), \rho_{v}(t)$. One can conclude that $e_{p}(t)$ and $e_{v}(t)$ not only respect their imposed funnels but also converge asymptotically to zero, without the need of arbitrarily small values for $\lim _{t \rightarrow \infty} \rho_{p}(t)$ and $\lim _{t \rightarrow \infty} \rho_{v}(t)$. This can be verified also by Fig. 2, which depicts the evolution of the transformed errors $\varepsilon_{p}(t), \varepsilon_{v}(t)$, $\forall t \in[0,55] \mathrm{sec}$, and shows their asymptotic convergence to zero. Finally, Figs. 3 and 4 illustrate the inputs $u(t)$ as well as the adaptation signal $\hat{d}(t), \forall t \in[0,55]$ sec. One can conclude the convergence of $\hat{d}(t)$ to a constant value as well as the boundedness of the control input $u(t)$, as was proved in the theoretical analysis.

\section{CONCLUSION AND FUTURE WORK}

This paper presents a novel control scheme that guarantees asymptotic stability subject to funnel constraints for a class of 2nd-order systems with unknown, nonlinear, and possibly discontinuous dynamics, from all initial conditions that satisfy the funnel constraints. We design a control protocol based on adaptive and discontinuous control methodologies and the correctness of the proposed scheme is independent from the control gain selection. Future efforts will be devoted towards extending the proposed scheme to more general systems with potential controllability relaxations.
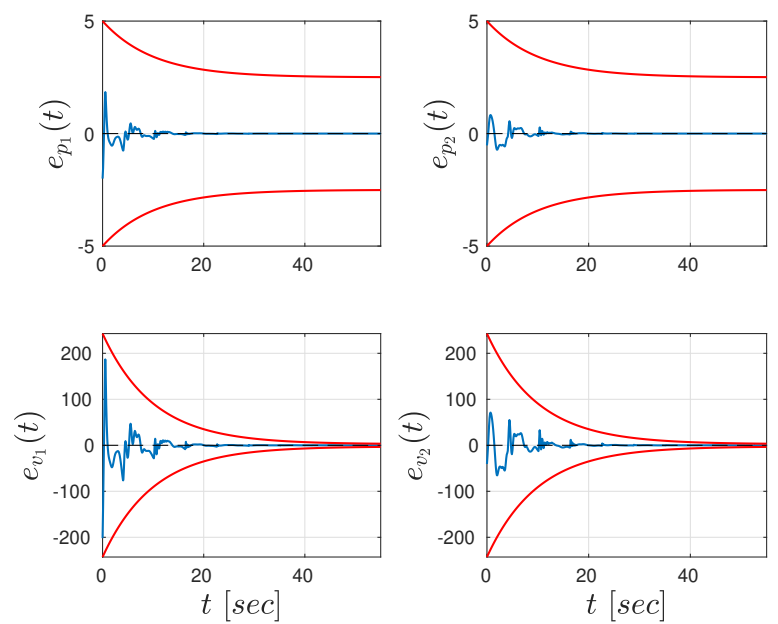

Fig. 1. The evolution of the errors $e_{p}(t)$ (top), $e_{v}(t)$ (bottom), depicted with blue, along with the performance functions $\rho_{p}(t), \rho_{v}(t)$, depicted with red, $\forall t \in[0,55]$ sec.
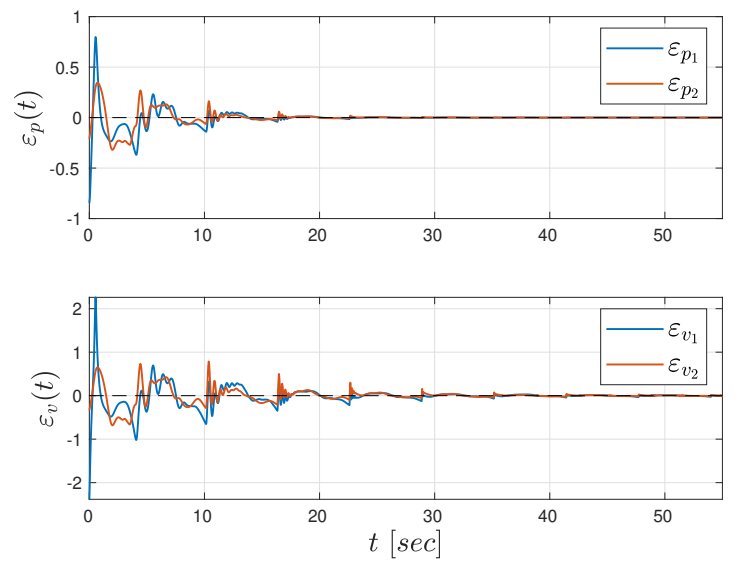

Fig. 2. The evolution of the transformed errors $\varepsilon_{p}(t), \varepsilon_{v}(t), \forall t \in[0,55]$ sec.

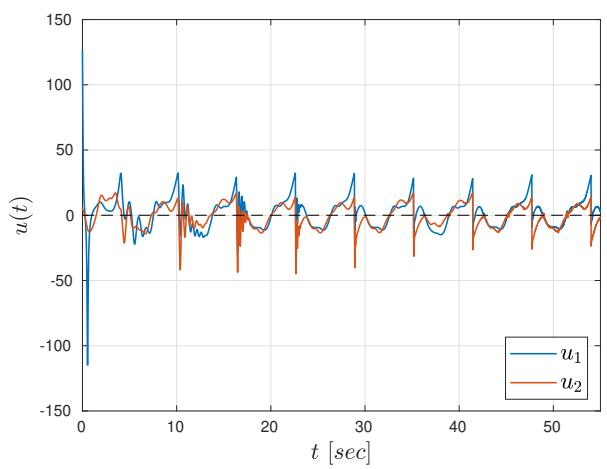

Fig. 3. The evolution of the control inputs $u(t)=\left[u_{1}(t), u_{2}(t)\right]^{\top}, \forall t \in$ $[0,55] \mathrm{sec}$. 


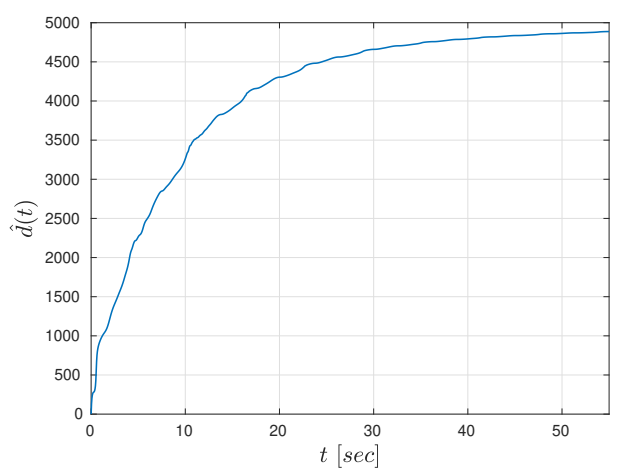

Fig. 4. The evolution of the adaptation signal $\hat{d}(t), \forall t \in[0,55]$ sec.

\section{REFERENCES}

[1] M. Krstic, I. Kanellakopoulos, and K. P., Nonlinear and adaptive control design. New York: Wiley, 1995.

[2] J. A. Farrell and M. M. Polycarpou, Adaptive approximation based control: unifying neural, fuzzy and traditional adaptive approximation approaches. John Wiley \& Sons, 2006, vol. 48.

[3] J. Chen, A. Behal, and D. M. Dawson, "Robust feedback control for a class of uncertain mimo nonlinear systems," IEEE Transactions on Automatic Control, vol. 53, no. 2, pp. 591-596, 2008.

[4] B. Xian, D. M. Dawson, M. S. de Queiroz, and J. Chen, "A continuous asymptotic tracking control strategy for uncertain nonlinear systems," Transactions on Automatic Control, vol. 49, no. 7, pp. 1206-1211, 2004.

[5] L. Wang, R. Ortega, H. Su, and Z. Liu, "Stabilization of nonlinear systems nonlinearly depending on fast time-varying parameters: an immersion and invariance approach," IEEE Transactions on Automatic Control, vol. 60, no. 2, pp. 559-564, 2015.

[6] A. Astolfi and R. Ortega, "Immersion and invariance: a new tool for stabilization and adaptive control of nonlinear systems," IEEE Transactions on Automatic control, vol. 48, no. 4, pp. 590-606, 2003.

[7] K. Zhang, Z. Wang, and A. Behal, "A continuous asymptotic tracking control strategy for a class of uncertain mimo nonlinear systems," Decision and Control (CDC), 2015 IEEE 54th Annual Conference on, pp. 6209-6214, 2015.

[8] R. Marino and P. Tomei, "An adaptive output feedback control for a class of nonlinear systems with time-varying parameters," IEEE Transactions on Automatic Control, vol. 44, no. 11, pp. 2190-2194, 1999.

[9] S. S. Ge and C. Wang, "Adaptive nn control of uncertain nonlinear pure-feedback systems," Automatica, vol. 38, no. 4, pp. 671-682, 2002.

[10] D. Wang and J. Huang, "Adaptive neural network control for a class of uncertain nonlinear systems in pure-feedback form," Automatica, vol. 38, no. 8, pp. 1365-1372, 2002.

[11] B.-J. Yang and A. J. Calise, "Adaptive control of a class of nonaffine systems using neural networks," IEEE Transactions on Neural Networks, vol. 18, no. 4, pp. 1149-1159, 2007.

[12] W.-Y. Wang, Y.-H. Chien, Y.-G. Leu, and T.-T. Lee, "Adaptive t-s fuzzy-neural modeling and control for general mimo unknown nonaffine nonlinear systems using projection update laws," Automatica, vol. 46, no. 5, pp. 852-863, 2010.

[13] C. P. Bechlioulis and G. A. Rovithakis, "Robust partial-state feedback prescribed performance control of cascade systems with unknown nonlinearities," IEEE Transactions on Automatic Control, vol. 56, no. 9, pp. 2224-2230, 2011.

[14] T. Berger, H. H. Lê, and T. Reis, "Funnel control for nonlinear systems with known strict relative degree," Automatica, vol. 87, pp. 345-357, 2018.
[15] A. Ilchmann, E. P. Ryan, and C. J. Sangwin, "Tracking with prescribed transient behaviour," ESAIM: Control, Optimisation and Calculus of Variations, vol. 7, pp. 471-493, 2002.

[16] C. M. Hackl, Non-identifier based adaptive control in mechatronics: Theory and Application. Springer, 2017, vol. 466.

[17] C. M. Hackl, N. Hopfe, A. Ilchmann, M. Mueller, and S. Trenn, "Funnel control for systems with relative degree two," SIAM Journal on Control and Optimization, vol. 51, no. 2, pp. 965-995, 2013.

[18] T. Berger and T. Reis, "Zero dynamics and funnel control for linear electrical circuits," Journal of the Franklin Institute, vol. 351, no. 11, pp. 5099-5132, 2014.

[19] Y. Karayiannidis and Z. Doulgeri, "Model-free robot joint position regulation and tracking with prescribed performance guarantees," Robotics and Autonomous Systems, vol. 60, no. 2, pp. 214-226, 2012.

[20] T. Berger, S. Otto, T. Reis, and R. Seifried, "Combined open-loop and funnel control for underactuated multibody systems," Nonlinear Dynamics, vol. 95, no. 3, pp. 1977-1998, 2019.

[21] C. K. Verginis, M. Mastellaro, and D. V. Dimarogonas, "Robust cooperative manipulation without force/torque measurements: Control design and experiments," Transactions on Control Systems Technology, 2019.

[22] L. Macellari, Y. Karayiannidis, and D. V. Dimarogonas, "Multi-agent second order average consensus with prescribed transient behavior," IEEE Transactions on Automatic Control, vol. 62, no. 10, pp. 52825288, 2017.

[23] C. K. Verginis, A. Nikou, and D. V. Dimarogonas, "Robust formation control in se for tree-graph structures with prescribed transient and steady state performance," Automatica, 2019.

[24] J. G. Lee and S. Trenn, "Asymptotic tracking via funnel control," IEEE Conference on Decision and Control, 2019.

[25] E. P. Ryan, C. J. Sangwin, and P. Townsend, "Controlled functional differential equations: approximate and exact asymptotic tracking with prescribed transient performance," ESAIM: Control, Optimisation and Calculus of Variations, vol. 15, no. 4, pp. 745-762, 2009.

[26] A. Ilchmann and E. P. Ryan, "Asymptotic tracking with prescribed transient behaviour for linear systems," International Journal of Control, vol. 79, no. 8, pp. 910-917, 2006.

[27] M. Dresscher and B. Jayawardhana, "Prescribing transient and asymptotic behaviour of lti systems with stochastic initial conditions," IFACPapersOnLine, vol. 50, no. 1, pp. 1822-1827, 2017.

[28] C. K. Verginis and D. V. Dimarogonas, "Asymptotic stability of uncertain lagrangian systems with prescribed transient response," IEEE Conference on Decision and Control, 2019.

[29] B. Paden and S. Sastry, "A calculus for computing filippov's differential inclusion with application to the variable structure control of robot manipulators," IEEE transactions on circuits and systems, vol. 34, no. 1, pp. 73-82, 1987.

[30] N. Fischer, R. Kamalapurkar, and W. E. Dixon, "Lasalle-yoshizawa corollaries for nonsmooth systems," IEEE Transactions on Automatic Control, vol. 58, no. 9, pp. 2333-2338, 2013.

[31] A. Ilchmann, E. P. Ryan, and P. Townsend, "Tracking with prescribed transient behavior for nonlinear systems of known relative degree," SIAM Journal on Control and Optimization, vol. 46, no. 1, pp. 210-230, 2007.

[32] C. P. Bechlioulis and G. A. Rovithakis, "Prescribed performance adaptive control for multi-input multi-output affine in the control nonlinear systems," IEEE Transactions on Automatic Control, vol. 55, no. 5, pp. 1220-1226, 2010.

[33] C. K. Verginis and D. V. Dimarogonas, "Timed abstractions for distributed cooperative manipulation," Autonomous Robots, vol. 42, no. 4, pp. 781-799, 2018.

[34] C. P. Bechlioulis and G. A. Rovithakis, "A low-complexity global approximation-free control scheme with prescribed performance for unknown pure feedback systems," Automatica, vol. 50, no. 4, pp. 12171226, 2014.

[35] B. Mltyagin, "The zero set of a real analytic function," arXiv:1512.07276v1, 2015.

[36] J. Cortes, "Discontinuous dynamical systems," IEEE control Systems, vol. 28, no. 3, 2008. 